\title{
GEODETIC APPLICATIONS OF GRAZING OCCULTATIONS
}

\section{W. DUNHAM}

USAF Aeronautical Chart and Information Center, St. Louis, Mo., U.S.A.

Up to about a century ago, occultations were often used to measure differences in geographical longitude. Extensive geodetic surveys, accurate chronometers, telegraphic communications, and later short-wave radio time services obviated the geodetic need for occultation observations, which are affected by geodetically severe uncertainties of stellar and lunar positions, lunar limb irregularities, and observers' personal equations. More sophisticated methods of observation would be needed before the Moon could again be useful to geodesy.

During this century, cinematography of Bailey's beads and the flash spectrum during total solar eclipses have been used to obtain the relative apparent position of the Sun and Moon to an accuracy which could be useful to geodesy. But observational opportunities were rare and few results of geodetic significance have been obtained.

Dr John O'Keefe and other workers at the US Army Topographic Command (then Army Map Service) investigated the possibility of using observations of occultations for geodetic purposes during the 1950's. They concluded that photoelectric timings of total occultations, made from widely separated positions on different datums (independent surveying networks), could be used to determine the positions of the datums with respect to each other if observed by the equal-limb-line method. A given occultation is observed from two or more stations on different datums, weather permitting. The stations are positioned so that the star will disappear (or reappear) at the same axis angle, or position angle measured with respect to the Moon's axis, to minimize uncertainties due to limb irregularities. That is, the stations are located on 'equal limb lines'. A fairly extensive observational program was undertaken during the late 1950's and early 1960's. The results obtained were not as accurate as expected, partly for reasons discussed below, and have, for the most part, been superseded by considerably more accurate results from artificial satellites. The grazing occultation method discussed below is potentially capable of results nearly as accurate as the most precise artificial satellite results, and could serve as a relatively inexpensive check of them.

During the past decade, many successful efforts have been made to visually observe grazing occultations near the northern and southern limits of the regions of visibility of occultations. In general, a team of observers establishes a series of stations perpendicular to the predicted limit and extending for about $2 \mathrm{~km}$ (Figure 1). Timings of the multiple disappearances and reappearances of the star are made at each station. These observed contacts can be used to infer information about the lunar profile within a few degrees of the axis angle of central graze (Figure 2). The contacts can be compared with the profile predicted by Watts' charts to obtain accurate relative celestial latitude of the star and Moon, and systematic shifts in the limb correction 


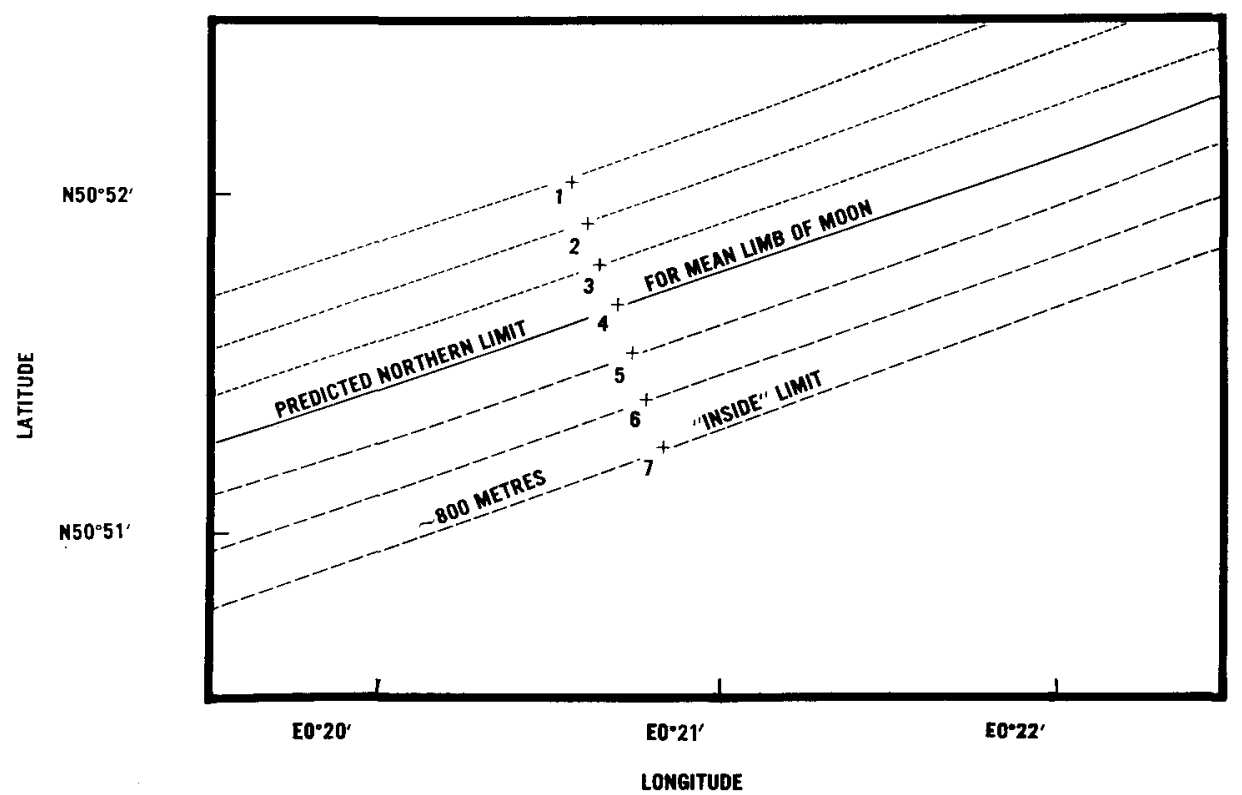

Fig. 1. Diagrammatic representation of the location of observers and the predicted limit.

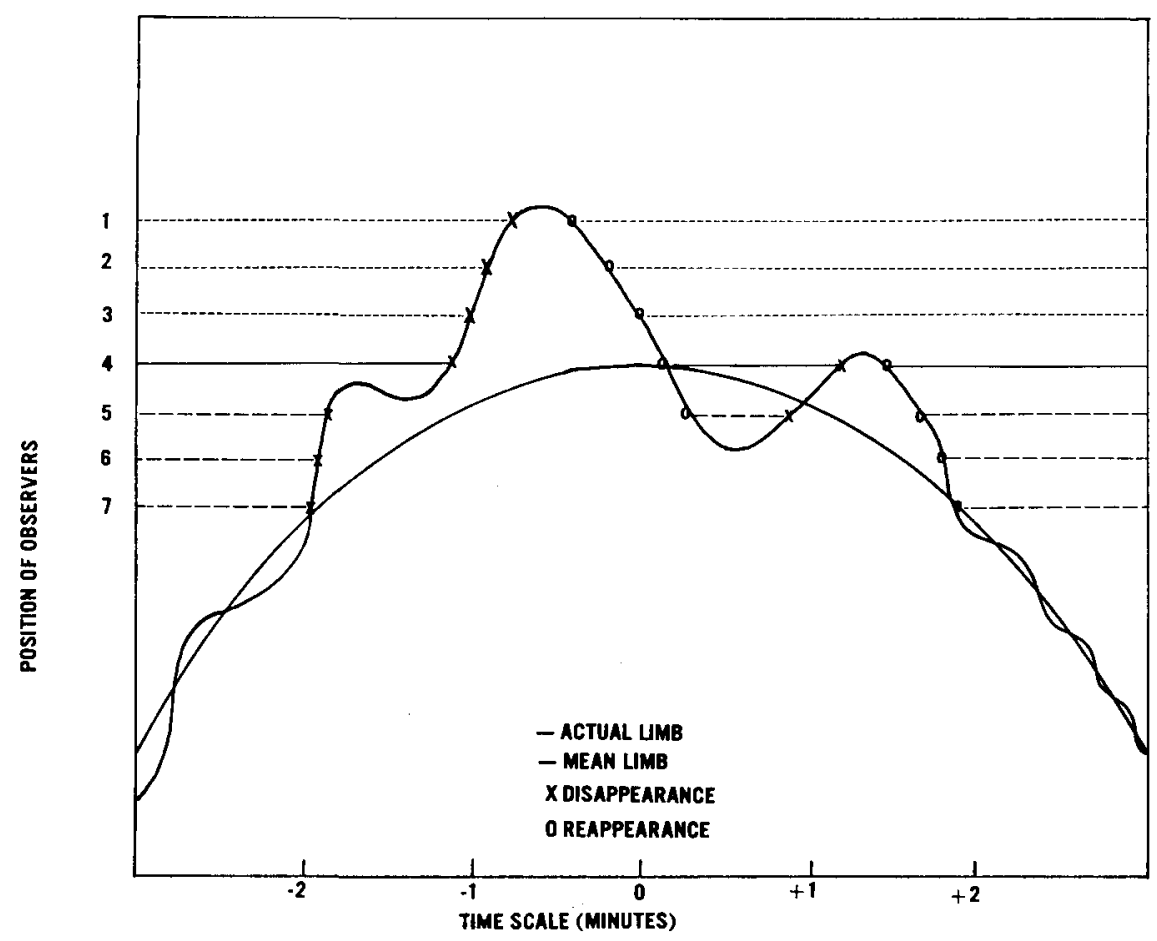

Fig. 2. Diagrammatic representation of the expected visibility of the star for each observer in Figure 1 


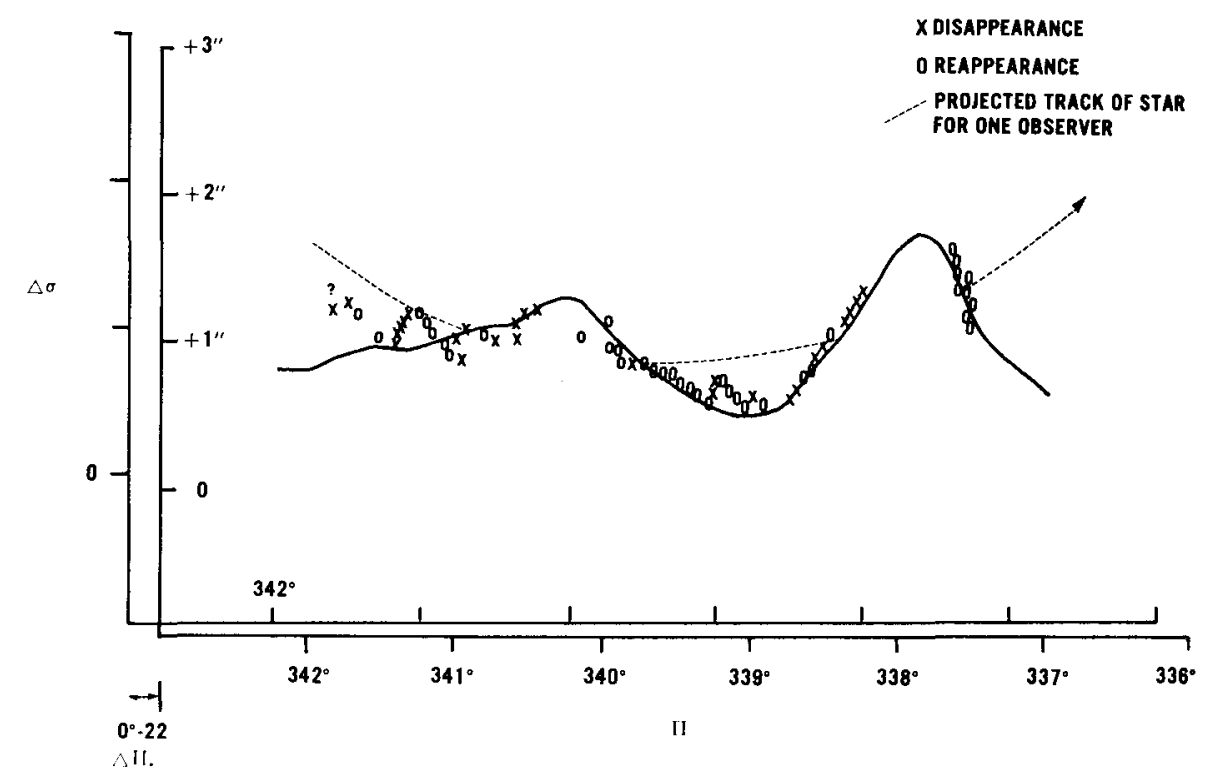

Fig. 3. Graze at the north limb of the Moon by $\delta$ Arietis observed from Indio, California on 1967 September 23.

data (Figure 3). Such investigations have been carried out by Dr Van Flandern and Messrs Morrison and Abileah. Observations of grazing occultations provide the most accurate data about the Moon's profile which we can obtain from the Earth's surface.

Lunar limb irregularities apparently caused most of the errors in the equal-limb-line observations. Grazing occultation observations can provide information about the lunar profile and, if suitably observed, can largely overcome the limb-irregularities problem, even if visual observations are used. The observations, made by observing teams in two or more widely-separated areas, can provide accurate data in the direction perpendicular to the occultation limit, and therefore can complement the equallimb-line observations, which give information only along the path. The observers in each team are located so that they will observe the same part of the Moon's profile. A relatively flat part of the profile predicted by Watt's charts should be selected to maximize the number of contacts by small-scale features. Since the axis angle of central graze will not be exactly the same at the separated locations, the observed paths of the star seen by the observers in the different groups will intersect in many places. If the Moon's actual limb passes very near some of these intersections, the observed contacts should coincide, that is, the observed times should occur at the same axis angle. If a few 'coincidences' are observed, but the axis angles of the contacts differ systematically between the two groups, an error in the location of one of the datums is implied. The amount of the geodetic shift can be inferred from the predicted profile and the timings made at adjacent stations. If axis angle coincidences occur where they are not predicted, the amount of shift needed to cause the observed coincidences would imply the geodetic shift. Of course, timings must be made ac- 
curately, preferably to \pm 0.2 , so that experienced occultation observers with good equipment should be used. Timing accuracies between stations can sometimes be inferred by timings made at steep parts of the profile, or by two observers at each station.

Due to the smooth terrain on many parts of the Moon and the grazing conditions, very high relative accuracies, around $20 \mathrm{~m}$ or even $10 \mathrm{~m}$ in the most favorable circumstances, seem possible. Since many points of the Moon's profile are observed, it is obvious when close coincidences occur, and by appropriate positioning of observers, they can be virtually guaranteed. In the equal-limb-line method, the lunar profile was not observed, and stations were often considerable distances from the equal limb lines due to geodetic uncertainties in the not-observed perpendicular direction and practical considerations. To compensate for these offsets from the equal limb line, a knowledge of the lunar slope in the vicinity of the observed contacts was needed. Since the photoelectric records were seldom of sufficient quality to determine the slope directly, Watts' charts were used to determine it. But the horizontal discrepancies now known to occur in the charts make the inference of slopes from them misleading.

The biggest uncertainty is due to the change in the profile caused by the different librations at the different sites. Along an occultation limit, and also to a high degree along an equal limb line, the latitude libration remains constant. All change is therefore in the longitude libration, equivalent to a rotation about the Moon's polar axis. The change in the profile is greatest at the lunar equator, where the geometry is most favorable for the equal-limb-line method, so that it is even possible that different lunar features will dominate at the same axis angle at two widely-separated locations. This is much less likely to occur during grazing occultations, which seldom occur more than $20^{\circ}$ from the Moon's poles. The small changes which do occur in the profile, which is itself largely observed, can be deduced from Watts' charts or, when circumstances permit it, from observation by a third team widely separated from, but located in the same datum as, one of the other teams.

Let $D$ be the difference in the predicted position angle of central graze at two widely-separated locations on an occultation limit. If $D$ is greater than about $1^{\circ}$, the component of the error in the position of the Moon with respect to the star perpendicular to the position angle of central graze becomes important. If $D$ is greater than $1^{\circ}$, a few good timings of the total occultation observed deep within the zone of visibility would usually be needed in order to reduce the relative position error enough for the graze observations to have geodetic significance. For larger values of $D$, coincidences become harder to arrange and timing errors become more important. The method cannot be used for $D$ larger than about $5^{\circ}$ unless, perhaps, extensive photoelectric observations are made of the shallow total occultation at one of the locations. Since data from a well-observed graze can be used to determine the star-Moon position to better than $50 \mathrm{~m}$ on the Earth's surface in some cases, it appears that grazes wellobserved from distant locations with large $D$ and no profile overlap might have some geodetic value. This is not so due to larger vertical discontinuities which occur in the limb correction data, recently noticed in reductions of graze and total observations. 


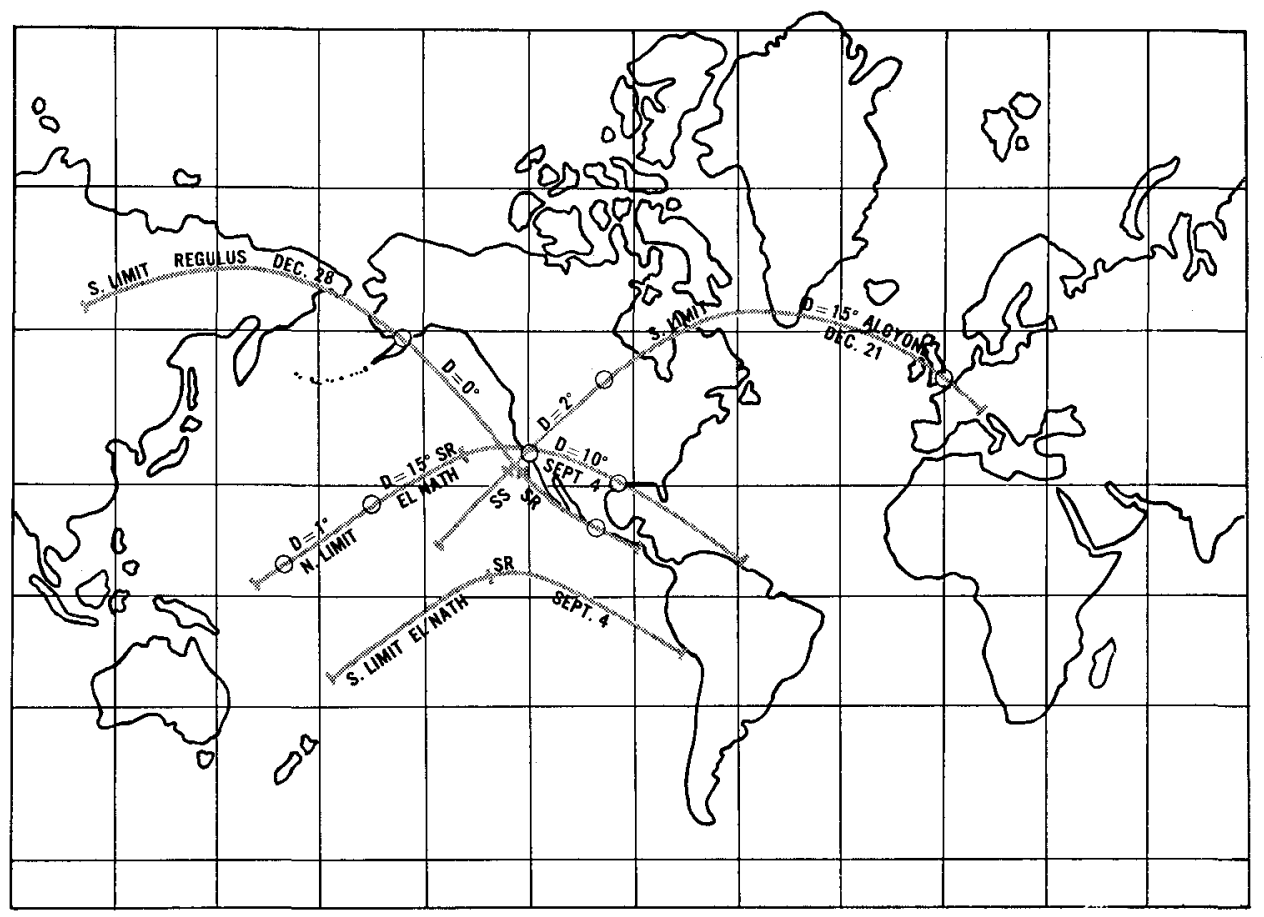

Fig. 4.

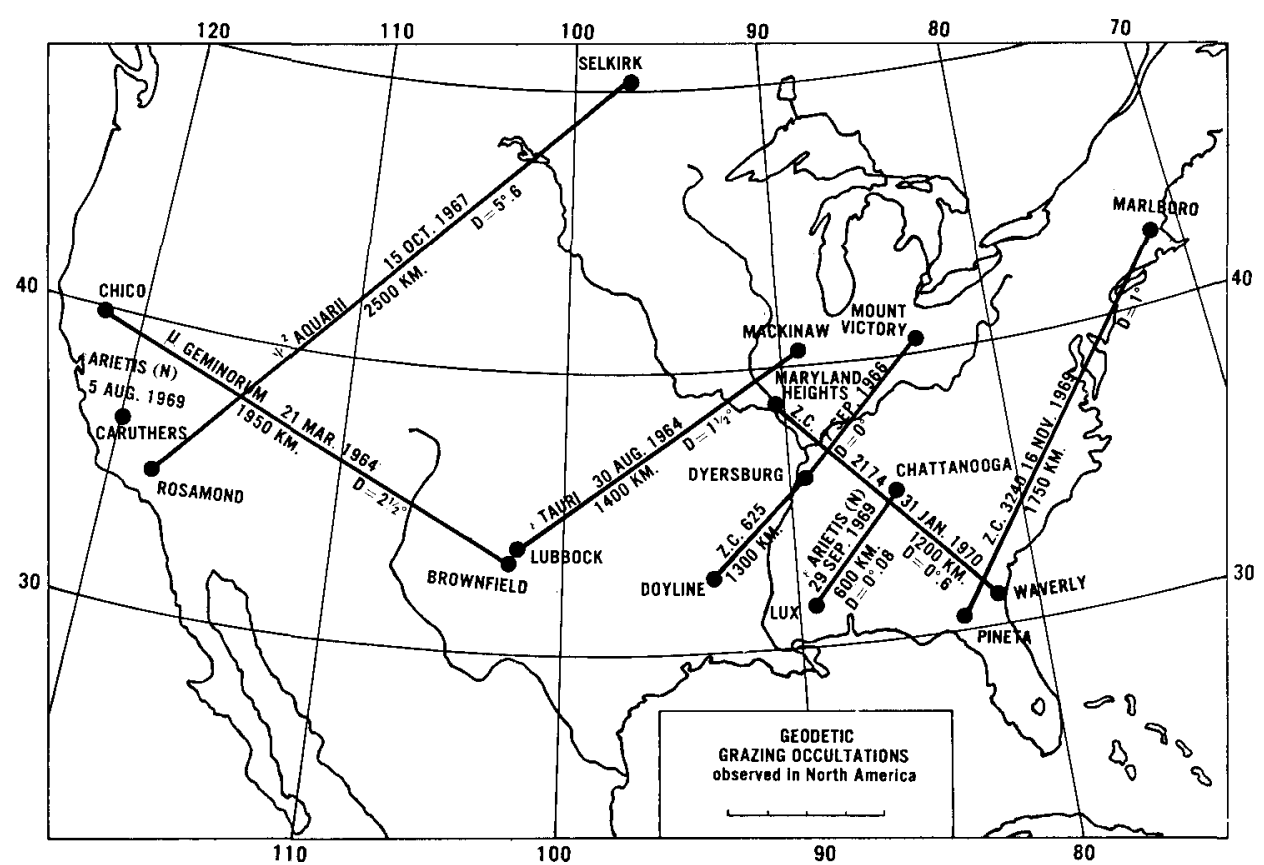

Fig. 5. 
Figure 4 shows some opportunities for making geodetic graze observations. Occultation limits are similar to total solar eclipse paths, either strongly bowed to the north or to the south, or $S$-shaped. The position angle of graze varies in approximately the same way that the bearing, or azimuth, of the limit varies, so that small $D$ 's occur between points along the limit where the direction of the limit is nearly the same as plotted on the Mercator projection. It follows, from the shape of limit curves, that the locations for geodetic graze observations must be at quite different geographic latitudes. Not shown on the map is another graze of Regulus which occurred on July 7th. The northern limit extended eastward from Virginia, then curved southward and finally eastward across Africa. An observer in Malawi, where the position angle of graze did not differ much from that in Virginia, attempted to observe the graze.

Since last summer, when the geodetic graze program was conceived at Aeronautical Chart and Information Center, several attempts have been made to coordinate observations from places separated by $1500 \mathrm{~km}$ or more along suitable graze paths within North America. This has been done to establish the feasibility and accuracy of the method, especially with regard to the change in the profile with longitude libration. Earlier graze observations made at widely-separated locations were examined for possible coincidences, but these grazes were observed with only astronomical goals in mind so that the spacing between observers in the teams was quite large. No coincidences were observed during the graze of $\mu$ Geminorum in March 1964, the first observed from widely-separated locations. No useful coincidences occurred during the graze of $\varepsilon$ Tauri in August 1964. Also, there were some timing problems and graze was on the bright limb, making observation difficult. The position for Z.C. 625 in 1966 was considerably in error, causing all observers to be too low on the profile, so that nothing of geodetic value could be obtained. A bright Moon, some timing problems, and a large $D$ value adversely affected the graze of $\psi^{2}$ Aquarii in 1967.

During June 27-28, 1969, a graze of Antares was observed from Ubaira, Brazil, and also by observers in Rhodesia. $D$ was very large but it showed that grazing occultations can be observed over intercontinental distances.

On November 16, 1969, a graze of 7 th magnitude Z.C. 3240 was observed by small teams in Florida and Massachusetts. The observations were not coordinated, but could be useful; they have not been reduced yet. The graze of Z.C. 2174 last January (1970) was coordinated and was quite successfully observed, but the data reduction is not yet complete.

The grazing occultation of the northern component of $\varepsilon$ Arietis, Z.C. 440, on September 29, 1969, was the first successfully-coordinated geodetic graze. A graze of the same star observed at about the same axis angles in California two sidereal months earlier, gave us confidence in the prediction for the September 29 event. The prediction for the earlier graze was itself accurate, but in general, observations of another graze of the same star a few months before a geodetic effort are helpful in refining the prediction, and necessary to avoid possible failures due to star position errors such as occurred with Z.C. 625 in 1966. This is especially true for the fainter stars. 


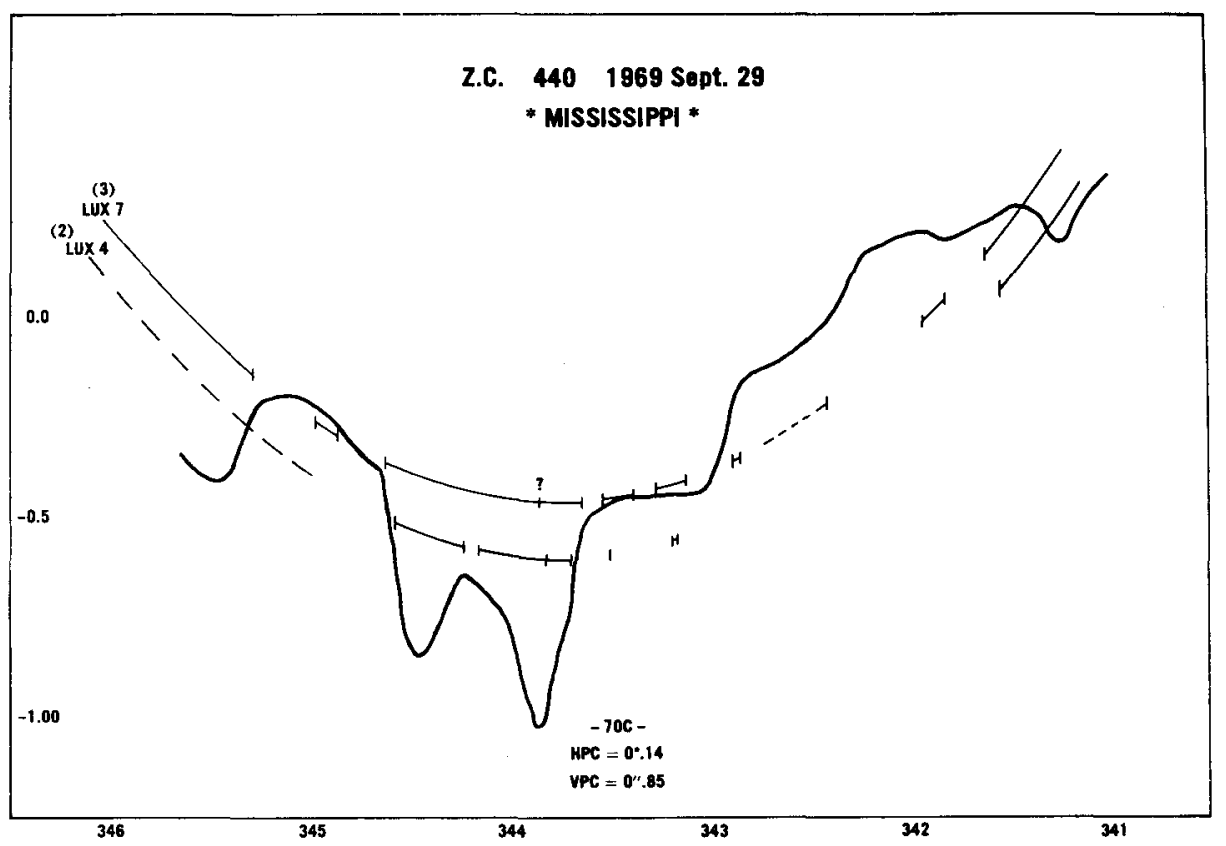

Fig. 6.

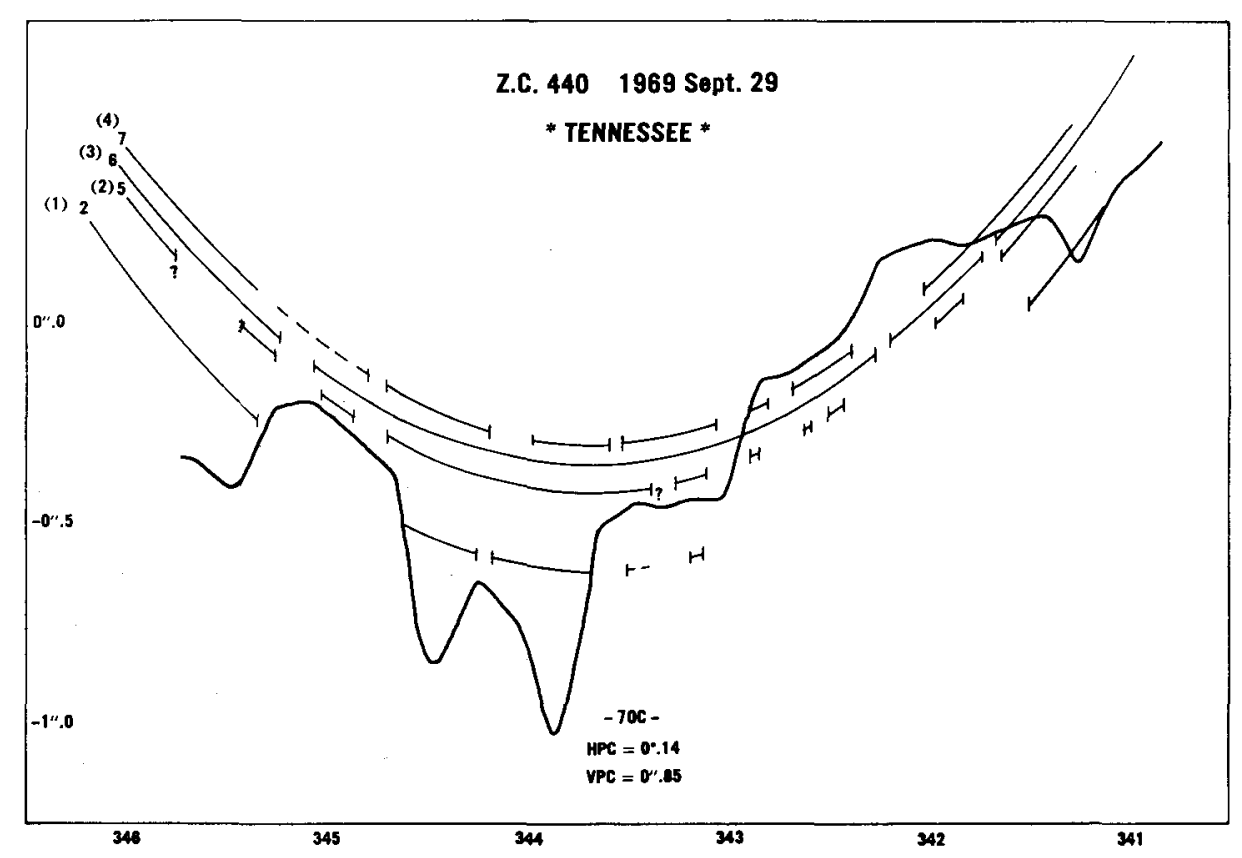

Fig. 7. 
Six expeditions, from New Orleans to Montreal, were ready for the graze on September 29. Clouds prevented observations north of West Virginia. Only the effort at Lux, Mississippi and near Chattanooga, Tennessee, were successful. Signals from buzzers and WWV time signals were recorded with tape recorders for timings. Unfortunately, the $600 \mathrm{~km}$ separation was not sufficient to derive any information about changes due to longitude libration.

Two stations were successful in the Mississippi expedition, numbers 3 and 2, manned by Mr Abileah and the speaker, respectively. The observations, represented by a solid curve when the star was visible, and the profile predicted from Watts' charts, are plotted in Figure 6. The reductions were done by $\mathrm{Mr}$ Abileah at the Computer Center of the University of Missouri at Kansas City, with some of the calculations done with Dr Van Flandern's reduction program. We intended to observe mainly the

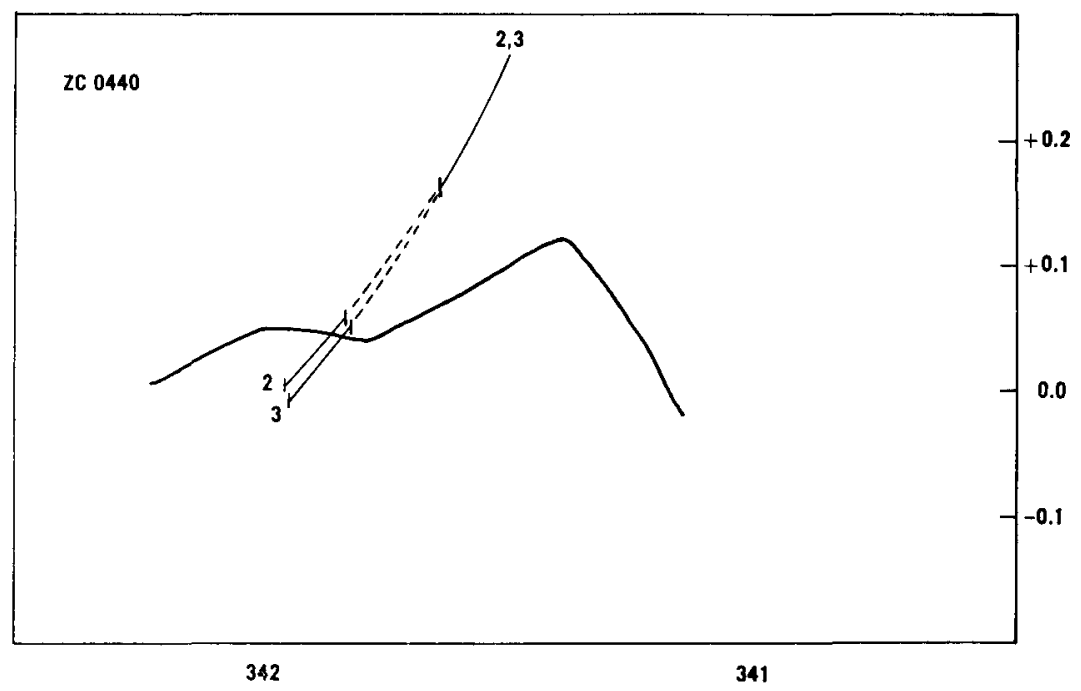

Fig. 8.

portion of the profile between axis angles $341^{\circ}$ and $344^{\circ}$, since the predicted paths of the star closely paralleled the surface. Timings were made from four stations in the Tennessee group, shown in Figure 7. Path intersections occurred at two points, one involving Mississippi 3 and Tennessee 2 at 341.6 and the other involving Mississippi 2 and Tennessee 1 at $344^{\circ} .15$. In both cases, the Moon's limb was nearby and coincidental events were observed.

In the first case, shown in detail in Figure 8, the coincidence occurred at the last reappearance at $341^{\circ} .65$. The apparent disagreement in the preceding disappearance time amounts to $0.3 \mathrm{sec}$ or 0.01 and is probably due to roundoff error, since the axis angles are computed to only 0.01 in the reduction.

The other coincidence is shown in Figure 9. Due to some observing difficulties, some of the events at axis angles less than $343^{\circ} .6$ were missed or poorly timed; these 


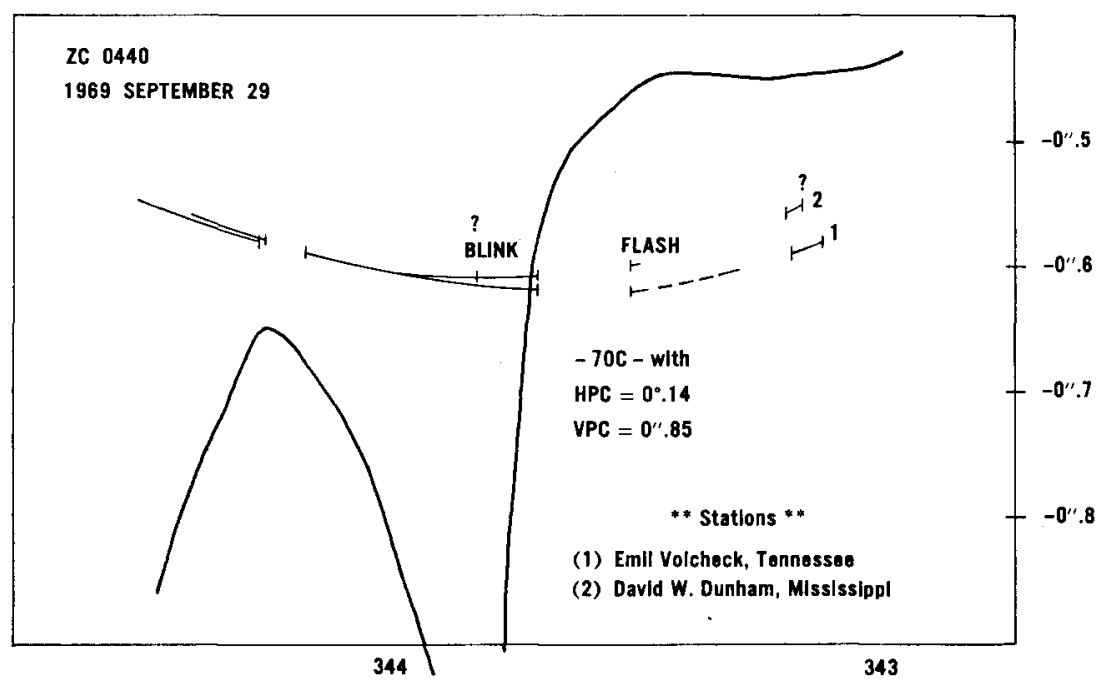

Fig. 9.

were noted by the observers in their original reports. The 'blink' at $343^{\circ} .83$ is probably spurious, caused by a moment of bad atmospheric seeing.

The top of the mountain at 344.2 occulted the star at both stations, for $2.7 \mathrm{sec}$ at station 1 in Tennessee and 3.4 seconds at station 2 in Mississippi. The coincidence occurred at the reappearance. If the paths-intersection is taken as the common reappearance point, the paths would be only 0.002 or $4 \mathrm{~m}$ apart at the disappearance, yet the time difference was $0.7 \mathrm{sec}$ ! This demonstrates the potential accuracies of the method, although the uncertainty in the vertical direction is actually about $15 \mathrm{~m}$ due to roundoff in the calculations (the heights are computed only to 0.01 ) and possible map errors.

One of the conclusions from this study is that the stations in each group should be more closely spaced, extending over a total distance of only a few hundred meters at most, with distance between stations not much larger than the accuracy desired in the final results (for instance, 20 or $25 \mathrm{~m}$ ). In this way, the fine structure of the profile in the vicinity of coincidences can be observed and observer timing errors and consistency can be better checked.

Somewhat better accuracies could be achieved with portable photoelectric equipment, but at rather greater cost. The accuracy would still be limited by the longitude libration profile changes and star position errors. However, it should be possible to trace the lunar profile for some distance away from the telescope location using fluctuations in the diffraction pattern. Also, coincidental photoelectric timings at steep features would give accurate data in the along-path direction, similar to the equal-limb-line method but more accurate since details of the profile in the region of interest can be observed. If a 'geodetic' graze path should happen to pass over two distant observatories with photoelectric capabilities, the opportunity should be utilized. 